\title{
Trends in Diabetes Care during the COVID-19 Outbreak in Japan: an Observational Study
}

J Gen Intern Med 36(5): 1460-2

DOI: $10.1007 /$ s11606-020-06413-w

(C) Society of General Internal Medicine 2021

\section{INTRODUCTION}

The COVID-19 pandemic has disrupted routine medical services worldwide. Physicians have been required to postpone routine outpatient visits of those with chronic diseases, including diabetes, to reduce the risk of COVID- 19 infection. ${ }^{1,2}$ Selfquarantine can pose a major threat to patients' access to primary care, which is necessary to prevent diabetes complications. Since uncontrolled diabetes can increase the risk of serious illness from COVID-19, ${ }^{1}$ reduced diabetes care may have significant public health consequences. However, little is known about how much the care for people with diabetes has reduced during this pandemic. We evaluated nationwide changes in diabetes care in outpatient settings during the COVID-19 outbreak in Japan.

\section{METHODS}

We used a de-identified hospital administrative database for Japanese acute care hospitals, built by Medical Data Vision Co., Ltd. (Tokyo, Japan). ${ }^{3}$ We analyzed hospital-level data on claims for each medical practice (identified by reimbursement codes) for 186 continuously observed hospitals during weeks 2-17 of 2019 and 2020, including the peak of the first wave of the COVID-19 outbreak (weeks 15-16 of 2020). We used this database because Japanese acute care hospitals offer large volume of routine outpatient ambulatory care as well as inpatient and emergency department care. ${ }^{4}$ We preliminarily found that this dataset covered 2,043,167 outpatient visits ( $5 \%$ of the nationwide hospital outpatient visits) in January 2019, according to the Hospital Report (a government-designated monthly survey that covers all hospitals nationwide).

We described the weekly numbers of claims for the following diabetes care procedures in outpatient settings: $\mathrm{HbAlc}$ test, serum creatinine test, urine protein test, fundus examination, diabetic foot care service, and diabetic kidney care service (details were in the Table 1 legend). We estimated the adjusted incidence rate ratios (aIRRs) by comparing counts of

Received July 31, 2020

Accepted December 8, 2020

Published online January 19, 2021 the procedures during the outbreak (weeks 9-17 of 2020, the period after February 25, when the Japanese government issued its first COVID-19 policy, referring to the suspension of large-scale gathering and recommendation for remote working) with those for weeks 2-17 of 2019 and weeks 2-8 of 2020 using a Poisson regression. Week-of-year and year dummy variables were included to adjust for seasonal and year-specific factors (difference-in-differences framework).

\section{RESULTS}

During weeks 9-17 of 2020, the weekly numbers of all the procedures for diabetes care declined compared to weeks 2-8 of 2020 (Fig. 1). The decline was statistically significant for all the procedures after adjusting for seasonal and year-specific factors. The number of procedures per week in weeks $2-8 \mathrm{vs}$. weeks $9-17$ of 2020 for $\mathrm{HbA} 1 \mathrm{c}$ tests decreased from 52,392 to 44,406 (crude rate reduction, $-15.2 \%$; aIRR, 0.86 ; $95 \%$ confidence interval [CI], $0.80-0.93 ; p<0.01)$, for serum creatinine tests from 143,064 to 122,806 ( $-14.2 \%$; aIRR, 0.87 ; $95 \%$ CI, $0.81-0.93 ; p<0.01$ ), for urine protein tests from 8329 to 7091 ( $-14.9 \%$; aIRR, 0.87 ; 95\%CI, 0.82-0.92; $p<0.01$ ), for fundus examinations from 24,568 to 21,096 ( $-14.1 \%$; aIRR, 0.83 ; 95\%CI, 0.76-0.91; $p<0.01)$, for diabetic foot care services from 369 to 309 ( $-16.4 \%$; aIRR, 0.85 ; 95\%CI, $0.74-0.97 ; p=0.01)$, and for diabetic kidney care services from 257 to 207 (- 19.4\%; aIRR, 0.83; 95\%CI, 0.74-0.94; $p<0.01$ ) (Table 1).

\section{DISCUSSION}

During the COVID-19 outbreak in Japan, the number of services for diabetes care significantly declined from the preceding weeks. The declines were also found for diabetic foot care service, which is recommended even during the pandemic. ${ }^{5}$ These findings suggest the possibility of long-term impacts on the development of diabetes complications. Efforts for constant follow-up of high-risk patients may be required to prevent diabetic vascular complications. ${ }^{1}$ Telemedicine may be promising for continuous diabetes care while reducing the risk of COVID-19 infection, ${ }^{6}$ given that the count of the reimbursement codes for telemedicine use was still small in Japan (accounting for $0.02 \%$ of all outpatient care services in our dataset). 
Table 1 Change in the Number of Diabetes Care Procedures During the COVID-19 Outbreak

\begin{tabular}{|c|c|c|c|c|c|c|c|}
\hline \multirow[t]{2}{*}{ Procedures $^{*}$} & \multicolumn{2}{|c|}{ No. of procedures per week* } & \multicolumn{2}{|c|}{$\begin{array}{l}\text { Difference between } \\
\text { weeks } 2-8 \text { and weeks } \\
9-17 \text { of } 2020\end{array}$} & \multicolumn{3}{|c|}{ Adjusted incidence rate ratio ${ }^{\dagger}$} \\
\hline & Weeks 2-8 of 2020 & Weeks 9-17 of 2020 & Counts & $\%$ change & Estimates & $95 \% \mathrm{CI}$ & $p$ values \\
\hline HbA1c test & 52,392 & 44,406 & -7986 & -15.2 & 0.86 & $0.80,0.93$ & $<0.001$ \\
\hline Serum creatinine test & 143,064 & 122,806 & $-20,257$ & -14.2 & 0.87 & $0.81,0.93$ & $<0.001$ \\
\hline Urine protein test & 8329 & 7091 & -1237 & -14.9 & 0.87 & $0.82,0.92$ & $<0.001$ \\
\hline Fundus examination & 24,568 & 21,096 & -3472 & -14.1 & 0.83 & $0.76,0.91$ & $<0.001$ \\
\hline Diabetic foot care service & 369 & 309 & -61 & -16.4 & 0.85 & $0.74,0.97$ & 0.014 \\
\hline Diabetic kidney care service & 257 & 207 & -50 & -19.4 & 0.83 & $0.74,0.94$ & 0.002 \\
\hline
\end{tabular}

CI, confidence interval

*The numbers of procedures were shown as a weekly average over the corresponding weeks

† Poisson regression was applied to estimate adjusted incidence rate ratio with week-of-year and year factors adjusted. Heteroscedasticity-robust standard errors were used for inference. $p<0.05$ was interpreted as statistically significant ( $R$ 3.6.2)

tReimbursement codes for the procedures were as follows: 160010010 (HbAlc test), 160019210 (serum creatinine test), 160000410 and 160004810 (urine protein test), $160081010,160081130,160203710$, 160203810, 160081550, and 160199310 (fundus examination), 113010010 (diabetic foot care service; foot care and related education provided by physicians and nurses for people with diabetes (PWD) with risks of diabetic gangrene, arteriosclerosis obliterans, or diabetic neuropathy), and 113013610 (diabetic kidney care service; clinical examinations and lifestyle education for PWD with diabetic nephropathy, conducted by physicians, nurses, and nutritionists)

Limitations of this study include the patient population, which covered only acute care hospitals (= general hospitals) but not clinics and thus might have more comorbidities compared to patients visiting clinics. Furthermore, some patients might be required to move to clinics for diabetes care during the outbreak to reduce COVID-19 infection risk at hospitals, which might explain part of our findings. Some of the procedures we evaluated were not specific to daily diabetes care (i.e., serum creatinine tests in emergency services). The clinical outcomes of decreased diabetes care remain unknown and warrant longer-term studies using patient-level data.

Ryo Ikesu, $M D^{1}$

Atsushi Miyawaki, $\mathrm{MD}, \mathrm{Ph} \mathrm{D}^{1}$

Takehiro Sugiyama, MD, $M S, P h D^{2,3,4}$

Masaki Nakamura, $B E^{5}$

Hideki Ninomiya, $M D^{6,7}$

Yasuki Kobayashi, $\mathrm{MD}, \mathrm{Ph} \mathrm{D}^{1}$

${ }^{1}$ Department of Public Health, Graduate School of Medicine, The University of Tokyo,

Tokyo, Japan

${ }^{2}$ Diabetes and Metabolism Information Center, Division of Health Services Research, National Center for Global Health and Medicine,

Tokyo, Japan

${ }^{3}$ Institute for Global Health Policy Research, Bureau of International Health Cooperation, National Center for Global Health and Medicine,

Tokyo, Japan

${ }^{4}$ Department of Health Services Research, Faculty of Medicine, University of Tsukuba,

Tsukuba, Ibaraki, Japan

${ }^{5}$ Medical Data Vision Co., Ltd.,

Tokyo, Japan
${ }^{6}$ Department of Health Policy and Management, Keio University,

Tokyo, Japan

${ }^{7}$ Datack, Inc.,

Tokyo, Japan

Corresponding Author: Atsushi Miyawaki, MD, PhD; Department of Public Health, Graduate School of Medicine, The University of Tokyo, Tokyo, Japan (e-mail: amiyawaki-tky@umin.ac.jp).

Author Contributions R.I. and A.M. designed the study and contributed to data acquisition, data analysis and interpretation, literature search, and writing of the manuscript. T.S. and Y.K. contributed to data analysis and interpretation and writing of the manuscript. M.N. and H.N. contributed to data collection and writing of the manuscript. All authors reviewed the manuscript and edited it for intellectual content and gave final approval for this version. A.M. is the guarantor of this work and, as such, had full access to all the data in the study and takes responsibility for the integrity of the data and the accuracy of the data analysis.

Funding The Medical Data Vision Co., Ltd. (Tokyo, Japan) provided the dataset used for this study to Dr. Atsushi Miyawaki in the form of labor service (non-financial support). The sponsor collected the data but did not have any other role in study design, analysis, interpretation of data, or writing the report.

\section{Compliance with Ethical Standards:}

The study was approved by the Institutional Review Board of the University of Tokyo (2020105NI).

Conflict of Interest: Mr. Masaki Nakamura is one of the board of directors in the Medical Data Vision Co., Ltd., and receives a salary from it outside this work. Dr. Hideki Ninomiya supports the Medical Data Vision Co., Ltd. in algorithm construction and receives payment outside this work. 

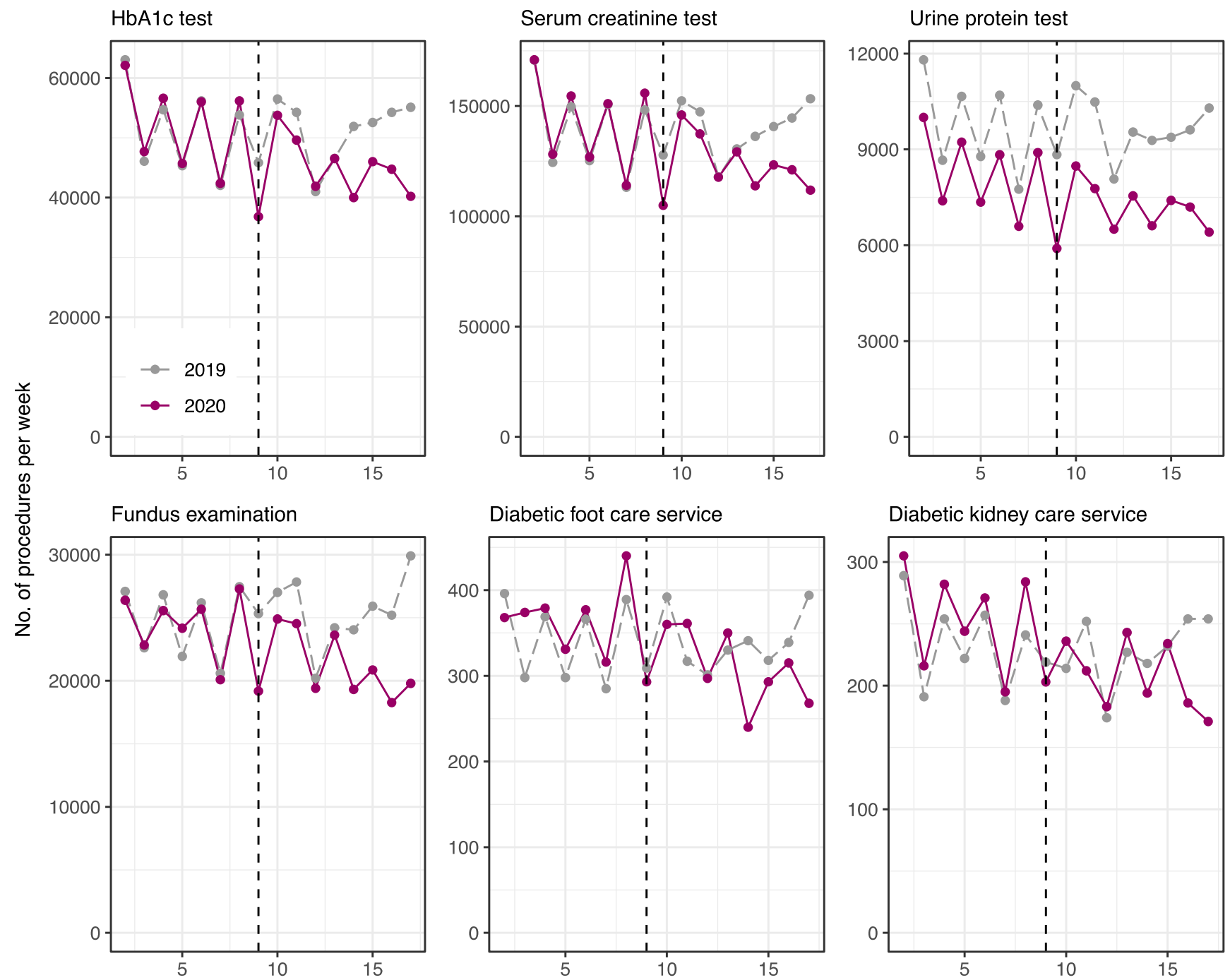

Diabetic foot care service

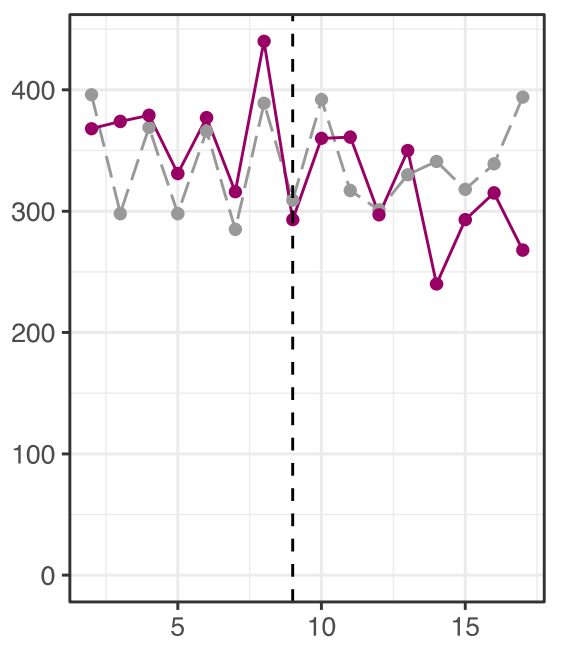

Diabetic kidney care service

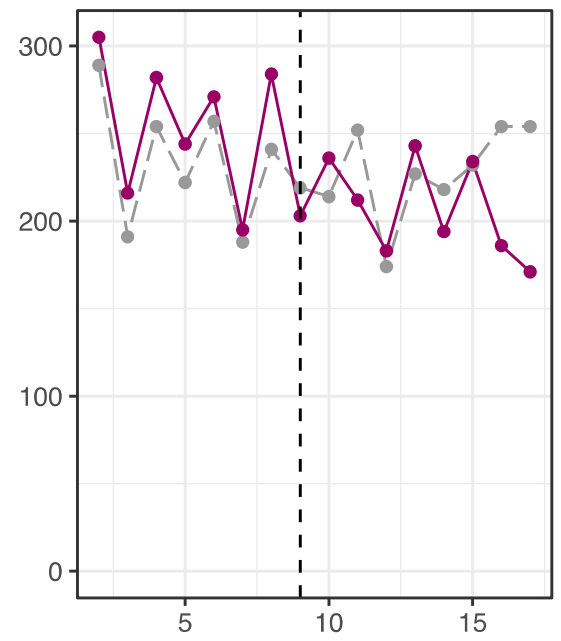

Week

\section{Events related to COVID-19 in 2020}

- The Japanese government issued its first COVID-19 policy (Feb 25; week 9 (dashed lines), referring to the suspension of large-scale gathering and recommendation for remote working), and the Ministry of Health, Labour and Welfare adopted this policy next week (week 10).

- $\quad$ World Health Organization declared the COVID-19 pandemic (Mar 11; week 11).

- The Japanese government declared a state of emergency for seven urban prefectures and asked people to stay at home (Apr 7; week 15). The statement was expanded to all over Japan (Apr 16; week 16).

- $\quad$ The number of new confirmed cases of COVID-19 culminated in weeks 15-16.

Fig. 1 Trends in the number of diabetes care procedures in outpatient settings for 186 Japanese hospitals in 2019 and 2020 . We identified each diabetic care procedure by using reimbursement codes for medical fee payments used throughout Japan. The number of procedures in week 1 (the year-end and New Year Holidays) was very small and thus not shown.

\section{REFERENCES}

1. Hartmann-Boyce J, Morris E, Goyder C, et al. Diabetes and COVID-19: risks, management, and learnings from other national disasters. Diabetes Care. 2020;43(8): 1695-1703. doi:https://doi.org/10.2337/dc20-1192

2. Wright A, Salazar A, Mirica M, Volk LA, Schiff GD. The invisible epidemic: neglected chronic disease management during COVID-19. J Gen Intern Med. Published online 2020. doi:https://doi.org/10.1007/ s11606-020-06025-4

3. Medical Data Vision Co., Ltd. Accessed July 22, 2020. https://en.mdv.co.jp/

4. Hashimoto $\mathbf{H}$, Ikegami $\mathbf{N}$, Shibuya $\mathbf{K}$, et al. Cost containment and quality of care in Japan: is there a trade-off? Lancet. 2011;378(9797):1174-1182. doi:https://doi.org/10.1016/S0140-6736(11)60987-2
5. NHS London Clinical Networks. Clinical strategy for service management of diabetic foot units during the COVID-19 pandemic. Accessed July 22, 2020. https://www.england.nhs.uk/london/wp-content/uploads/sites/ 8/2020/04/5.-Covid-19-Clinical-Strategy-for-MDFTs-Crib-Sheet02042020.pdf

6. Garg SK, Rodbard D, Hirsch IB, Forlenza GP. Managing new-onset type 1 diabetes during the COVID-19 pandemic: challenges and opportunities. Diabetes Technol Ther. 2020;22(6):431-439. doi:https://doi.org/10.1089/ dia.2020.0161

Publisher's Note: Springer Nature remains neutral with regard to jurisdictional claims in published maps and institutional affiliations. 